\title{
КОГНИТИВНОЕ УПРАВЛЕНИЕ ИНТЕЛЛЕКТУАЛЬНЫМИ БИЗНЕС-СИСТЕМАМИ: КОНЦЕПТУАЛЬНЫЕ И ТЕХНОЛОГИЧЕСКИЕ ОСНОВЫ
}

\author{
Гернет Н.Д., Лисицкий В.Л.
}

В работе предлагается предметная технология когнитивного управления интеллектуальными бизнес системами, функционирующими в условиях интенсивного формирования информационного общества. В качестве основы когнитивного управления используется технология ситуационного управления. Рассмотрены особенности этапов когнитивного управления. Разработана прочееура идентификации и разрешения проблемных ситуаций, возникающих на этапе реализации стратегии интеллектуальной бизнес системы.

DOI: $10.20537 /$ mce2020econ05

Введение. На современном этапе развития мировой системы происходит интенсивное формирование экономики знаний - высшего этапа постиндустриальной инновационной экономики, характеризуемого становлением информационного общества или общества знаний. Этот процесс сопровождается созданием глобальных сетевых технологий, интеллектуальных систем поддержки принятия решений, созданием когнитивных бизнес систем, появлением электронного бизнеса, управления знаниями, когнитивного управления бизнесом и т.п. Переход от «материальной» экономики, основанной на физическом труде, механизации, автоматизации, к «интеллектуальной» экономике, характеризуемой значительным повышением роли человеческого и социального капитала, инноваций, информации, креативного труда, творческой деятельности в различных сферах, рассматривается как глобальный структурный сдвиг, который, охватывая все сферы и отрасли постиндустриальной экономики, изменяет ее масштаб, динамику, внутреннее содержание. В этих условиях возникла необходимость пересмотра старых методов управления и организации. Экономика знаний потребовала не только управления материальными активами, ресурсами, себестоимо- 
стью, но также и управления нематериальными активами, интеллектуальным капиталом. В условиях экономики знаний для интеллектуальных бизнес систем (ИБС) характерно следующее $[1,2]$ :

- информация и знания становятся ресурсом и движущей силой развития;

- рынок информации и знаний (рынок интеллектуальной продукции) становится фактором производства;

- удельный вес отраслей знаний и информации растет быстрыми темпами;

- конкурентоспособность определяется развитой информационной инфраструктурой;

- развитие и внедрение современных информационных технологий существенно меняет модели труда, обучения, жизни, отдыха персонала.

ИБС как сложная система представляет собой конечное множество взаимодействующих структурных элементов, образующих определенную организационную структуру, предназначенную для активного достижения зафиксированной глобальной цели с максимальной эффективностью. ИБС обладает человеческим, производственным, интеллектуальным капиталом, функциональными подсистемами, множеством бизнес процессов (основных, обеспечивающих, бизнес процессов управления и развития). Ведущим компонентом ИБС является человеческий капитал, определяемый лицами, принимающими решение на различных уровнях, коллективом ИБС, персоналом. ИБС - это не только «умные» технологии, но также персонал, который обучен и мотивирован на их применение. В ИБС обеспечивается подвижность и гибкость бизнес процессов, использование более глубокого интеллекта на всех уровнях ИБС, быстрого и масштабного внедрения инноваций. В связи с этим для управления ИБС целесообразно использовать методы когнитивного управления, основанного на знаниях и учитывающего специфические особенности действующих технологических и человеческих ограничений. В качестве основы для когнитивного управления ИБС целесообразно использовать технологию ситуационного управления. Она предполагает принятие управленческих решений не в установленные сроки, а по мере обнаружения проблемных ситуаций (ПС), определяющих проблемную управленческую ситуацию - субъективную оценку характеристик ИБС и внешней среды, связей между ними в те- 
кущее время, зависящих от прошлых событий, развивающихся в пространстве и времени. Различные, возникающие ПС требуют различных типов знаний, различных подходов к их разрешению. С учетом этого ситуационное управление - это, прежде всего, искусство определения и оценки ПС, выбора эффективных управляющих воздействий, адекватным образом отвечающих возникающей ПС [3]. В этом случае пригодность различных методов управления определяется ПС, нет единого «лучшего» способа управления для различных ПС. Самым эффективным методом в конкретной ПС является такой, который более всего соответствует возникшей ПС. Ситуационное управление подчеркивает значимость ситуационного мышления, которое интегрирует различные частные подходы, увязывает конкретные приемы и концепции с определенными ПС для их успешного разрешения [4].

2. Модель процесса когнитивного управления. Цикл процесса когнитивного управления (т.е. управления, основанного на знаниях, интуиции, креативности) состоит из следующих этапов.

Этап 1. Определение (коррекция) видения будущего ИБС, которое может быть достигнуто при самых благоприятных условиях. Видение является эффективным средством мотивации персонала ИБС. Оно сплачивает, объединяет деятельность людей в едином заданном направлении.

Этап 2. Определение (коррекция) миссии ИБС, задающей глобальную цель, которая должна быть достигнута на фиксированном временном интервале. Миссия является базисом плановых решений, создает уверенность, что ИБС преследует непротиворечивые, ясные, сравнимые цели, сосредотачивает внимание персонала на выбранном направлении, сплачивает его действия.

Этап 3. Построение дерева целей ИБС. Дерево целей представляет собой упорядоченное семейство целей по ключевым пространствам деятельности ИБС (положение на рынке, инновации, производительность, ресурсы, прибыльность, персонал, менеджмент и т.п.) [5].

Этап 4. Оценка стратегического потенциала, стратегического климата, стратегической позиции ИБС. Оценка способности ИБС реализовать дерево целей.

Этап 5. Формирование стратегии реализации дерева целей ИБС, определяющей долгосрочное, качественно определенное направление развития ИБС. Стратегия ИБС представляет собой конечное множество 
$S=\left\{S_{j}, \mathrm{j}=\overline{1, g}\right\} \quad$ компонент $S_{j} \in S$, которое определяется суммой $S=S^{1} \cup S^{2} \cup S^{3}$, где $S^{1} \subset S, S^{2} \subset S, S^{3} \subset S, S^{1}$ - множество компонент корпоративной стратегии ИБС, $S^{2}-$ множество компонент конкурентной стратегии ИБС, $S^{3}-$ множество компонент функциональных стратегий ИБС. Если $F=\left\{f_{i}, i=\overline{1, P}\right\}-$ множество функциональных зон ИБС $f_{i}, P$ - количество выделенных функциональных зон, то $S^{3}=S_{31} \cup \cdots \cup S_{3 j} \cup \cdots \cup S_{3 q}$, где $g_{0}=\sum_{j=1}^{g} g_{j}, \quad g_{j}$ - мощность множества $S_{3 j}, S_{3 j}$ - множество компонент стратегии $j$-ой функциональной зоны. Представленная морфология стратегии ИБС, взаимосвязи между ее компонентами, определяющие дискретную структуру стратегии, существенно затрудняют процесс ее реализации $[6,7]$.

Этап 6. Управление реализацией стратегии ИБС.

Управление реализацией стратегии компенсирует негативные последствия, которые могут возникнуть при осуществлении стратегии по причине имеющихся у нее недостатков либо в силу появления во внешней среде неожиданных изменений, существенно меняющих стратегический климат. Управление реализацией стратегии ИБС включает осуществление контроля за реализацией стратегии и систематическую корректировку стратегии. При контроле важна не столько высокая точность выполнения отдельных работ по реализации стратегии, сколько выяснение того, насколько реализуемая стратегия соответствует текущим внутренним и внешним условиям функционирования ИБС и в какой степени фактическое направление развития способно привести ИБС в целевое состояние. Если выявленные отклонения создают существенную угрозу достижению целевого состояния, то говорят о возникновении проблемной ситуации (ПС) [6].

Процесс идентификации и разрешения ПС представляет собой процедуру, содержащую следующие этапы.

Этап 1. Получение информации о сложившейся текущей ситуации и определение фактического направления развития ИБС, задаваемого вектором $\vec{x}_{\phi}(t)=\left(x_{\phi}^{1}(t), \cdots, x_{\phi}^{j}(t), \cdots, x_{\phi}^{n}(t)\right)$, где $x_{\phi}^{j}(t)$ - численное значение ј-го стратегического показателя развития, $\mathrm{t}$ - текущий момент времени. Если $\vec{x}_{n}(t)=\left(x_{n}^{1}(t), \cdots, x_{n}^{j}(t), \cdots, x_{n}^{n}(t)\right)$ - плановое направле- 
ние развития, то $\cos \alpha(\mathrm{t})=\left(\vec{x}_{n}(t), \vec{x}_{\phi}(t)\right) /\left|\vec{x}_{n}(t)\right| \cdot\left|\vec{x}_{\phi}(t)\right|$ определяет угол $\alpha(\mathrm{t})$ между плановым и фактическим направлениями развития ИБС. В случае, когда нарушается неравенство $\cos \alpha(\mathrm{t}) \geq 1-\varepsilon$, где $\varepsilon-$ допустимая погрешность, что говорит о возникновении ПС и формируется иерархия для определения глобальных приоритетов функциональных зон $f_{i} \in F$ и компонент $S_{j} \in S$ стратегии ИБС. Вершина иерархии определяет фокус проблемы - увеличение разрыва между плановым и фактическим направлениями развития. Второй уровень иерархии определяет главные цели развития ИБС. На третьем уровне располагаются функциональные зоны ИБС. На четвертом (нижнем) уровне иерархии находятся компоненты $S_{j}$ стратегии ИБС.

Этап 2. Заполнение экспертами матриц попарных сравнений для всех вершин иерархии, определение нормированных локальных приоритетов главных целей, функциональных зон, компонент стратегии.

Этап 3. Определение глобальных приоритетов (значимостей) $\alpha_{i}-$ функциональных зон $f_{i}$ и $\beta_{j}$-компонент стратегии $S_{j}$.

Для выделения значимых $f_{i}$ их ранжируют по степени убывания их глобальных приоритетов и формируют множество $F_{0}$ из первых элементов полученного ряда. $F_{0}=\left\{f_{s_{i}}: \sum_{i=1}^{P_{0}} S_{i}=0,8 \pm \varepsilon\right\}$, где $\varepsilon$ - допустимая погрешность, $P_{0}$ - мощность множества $F_{0}$. Аналогично выделяют значимые компоненты $S_{j}$ стратегии. Для этого $S_{j}$ ранжируют по степени убывания их приоритетов $\beta_{j}$ и формируют множество $S_{0}$ из первых элементов полученного ряда $S_{0}=\left\{S_{l_{j}}: \sum_{j=1}^{g_{0}} \beta_{l_{j}}=0,8 \pm \varepsilon\right\}$, где $g_{0}-$ мощность множества $S_{0}$. После чего значимости $\alpha_{s_{i}}$ для $f_{s_{i}} \in F_{0}, \beta_{l_{j}}$ для $S_{l_{j}} \in S_{0}$ нормируют: $\quad \alpha_{s_{j}}^{0}=\alpha_{s_{i}} / \sum_{i=1}^{p_{0}} \alpha_{s_{i}}, \quad \beta_{l_{j}}^{0}=\beta_{l_{j}} / \sum_{j=1}^{g_{0}} \beta_{l_{j}}$. Двудольный граф $G_{0}$, определенный на множестве вершин $F_{0}$ и $S_{0}$, задает структуру проблемосодержащей среды. Ребра графа $G$ определяются условиями: 
- $<f_{s_{i}}, S_{l_{j}}>\in R_{0} \subset F_{0} \times S_{0}, f_{s_{i}} \in F_{0}, \quad S_{l_{j}} \in S_{0} ;$

- функциональное звено $f_{s_{i}}$ участвует в реализации компоненты $S_{l_{j}}, f_{s_{i}} \in F_{0}, S_{l_{j}} \in S_{0}$.

Этап 4. Формирование матрицы $A_{0}^{\phi}$ для двудольного орграфа с множеством вершин $F_{0}^{\phi}, \mathrm{S}_{0}^{\phi}$. Элементы матрицы $A_{0}^{\phi}$ определяются формулой

$$
a_{i j}^{0}=\left\{\begin{array}{l}
1, \quad<f_{s_{i}} S_{l_{j}}>\in R_{0} \\
0, \quad<f_{s_{i}} S_{l_{j}}>\notin R_{0}
\end{array}, \text { где } i=\overline{1, p}, \mathrm{j}=\overline{1, \mathrm{~g}} .\right.
$$

Этап 5. Поиск в базе прецедентов класса $T_{q}$, эталонная ПС которого, определяемая матрицей $A_{0}^{\phi}$, наиболее близка к фактической ПС.

Если имеется две проблемные ситуации $\Pi C^{k}$ и $\Pi C^{l}$, то расстояние между ними $\mathrm{d}\left(\Pi C^{k}, \Pi C^{l}\right)$ определяется расстоянием между матрицами $A_{0}^{k}=\left(a_{i j}^{0 k}\right) \quad$ и $\quad A_{0}^{l}=\left(a_{i j}^{0 l}\right), \quad i=\overline{1, p}, \mathrm{j}=\overline{1, \mathrm{~g}} \quad$ и $\quad$ равно $d\left(\Pi C^{k}, \Pi C^{l}\right)=\sqrt{\sum_{i=1}^{p} \sum_{j=1}^{g}\left(a_{i j}^{0 k}-a_{i j}^{0 l}\right)^{2}}$.

В случае, когда расстояние $d\left(\Pi C^{k}, \Pi C^{l}\right)$ меньше заданного порога $\delta, \delta>0$, говорят о принадлежности $\Pi C^{k}$ и $\Pi C^{l}$ к одному классу $T_{q}$ проблемных ситуаций, соответствующих некоторому прецеденту с номером $q$, $q=\overline{1, H} . H$ - число прецедентов, зафиксированных в базе прецедентов.

Если прецедент $q$ определяет детерминированные ПС, то их матрицы смежности близки и класс $\Pi C^{r} \in T_{q}$ можно представить эталонной $\Pi C^{\ni}$ с матрицей $A_{0}^{\ni}$, имеющей элементы $a_{i j}^{0 \ni}$

$$
a_{i j}^{0 \ni}=\left\{\begin{array}{l}
1,\left(\sum_{\Pi C^{r} \in T_{q}} a_{i j}^{0 r}\right) / H_{q} \geq h-\varepsilon \\
0,\left(\sum_{\Pi C^{r} \in T_{q}} a_{i j}^{0 r}\right) / H_{q}<h-\varepsilon
\end{array},\right.
$$

где $H_{q}$ - мощность $T_{q}, h$ - заданный пороговый уровень, $\varepsilon$ - допустимая погрешность. 
Для детерминированных ПС величина порога $1 \geq h \geq 0.8$. Для недетерминированных ПС величина порога $h$ снижается.

Этап 6. Фиксация найденного прецедента. Формирование на его основе возможных мероприятий по разрешению возникшей детерминированной ПС. Адаптация управленческих решений.

Если для текущей ПС аналога не обнаружено, то она либо определяет неизвестный класс ПС, либо является индетерминированной. В обоих случаях необходим когнитивный анализ ПС.

3. Результаты. Основной задачей когнитивного анализа ПС является выбор модели управления, обеспечивающий разрешение ПС, предотвращение нежелательных последствий ее развития, уменьшающей величину разрыва между плановым и фактическим направлениями развития ИБС. Предлагаемая технология решения задачи включает в себя следующие шаги.

1. Систематический сбор, систематизация, системный анализ существующей статистической и качественной информации и знаний: о реализации стратегий; о всех известных прецедентах; о принятых управленческих решениях по разрешению ПС; имеющихся рекомендациях и другом опыте; о результатах, проведенных и проводимых научных исследований по идентификации, распознаванию, разрешению ПС для подобных прецедентов и т.п. [9].

2. Построение проблемного поля текущей ПС. Как правило, ПС не существует в чистом виде. Все возникающие ПС взаимосвязаны. К их разрешению следует подходить холистически, с единых системных позиций, определяемых фокусом проблемы. В качестве основы для построения проблемного поля $\Phi_{\text {ПС }}$ задается множество этих проблемных ситуаций $\Phi_{\Pi С}=\left\{E_{i j}:<f_{i}, \mathrm{~s}_{\mathrm{j}}>\in \mathrm{R}_{0}^{\phi}\right\}$.

3. Структурирование проблемного поля текущей ПС. Для осуществления структуризации $\Phi_{П С}$ группа экспертов на основе значений показателей эффективности деятельности ИБС, показателей использования стратегического потенциала ИБС, имеющихся оценок стратегического климата ИБС, значений показателей, характеризующих внутриорганизационные процессы определяет пары концептов $<E^{k}, E^{l}>, E^{k}, E^{l} \in \Phi_{П С}$ между которыми существует каузальная связь: наличие проблемы с $k$ номером вызывает появление проблемы с номером $l$. Бинарное отношение 
$R_{0 \Pi C} \subset \Phi_{\Pi C} \times \Phi_{\Pi C}$ задает орграф $G_{\Pi C}$, матрица смежности которого позволяет представить $\Phi_{П С}$ в виде суммы $\Phi_{\Pi C}=\Phi_{\Pi C}^{1} \cup \Phi_{\Pi C}^{2} \cup \Phi_{\Pi C}^{3}$. Если при построении $R_{0 П C}$ обеспечена ацикличность орграфа $G_{\Pi C}$, то $\Phi_{\text {ПС }}^{1}$ - множество корневых проблем, $\Phi_{П С}^{2}$ - множество результирующих проблем, $\Phi_{\Pi C}^{3}$ множество промежуточных проблем. Множество $\Phi_{\text {IІ }}^{1}$ определяет множество корневых проблем, с разрешением которых устраняются нежелательные последствия развития текущей ПС, обуславливающие увеличение разрыва между плановым и фактическим развитием ИБС [10].

4. Формирование модели управления разрешением текущей ПС. При формировании модели управления используется сценарный подход. В этом случае сценарий преодоления ПС представляется в виде неформализованного, многоальтернативного прогноза, описывающего возможную последовательность действий, событий для того, чтобы показать, как, исходя из сложившегося положения дел, ИБС может шаг за шагом преодолевать текущую ПС. Сценарий определяет механизм координации, взаимной увязки как в пространстве, так и во времени целей, поведения функциональных зон в процессе разрешением текущей ПС и сокращения разрыва между плановым и фактическим направлениями развития ИБС. Для разрешения текущей ПС необходимо разрешить все корневые проблемы из $\Phi_{П С}^{1}$. При формировании сценария разрешения отдельной проблемы из $\Phi_{П С}^{1}$ применяются средства когнитивного моделирования, предполагающего построение когнитивной карты (КК). КК позволяет эффективно исследовать поведение сложных систем, не поддающихся точному математическому анализу. Так как проблема из $\Phi_{\text {ПС }}^{1}$ определяется концептом $<f_{i}, \mathrm{~S}_{\mathrm{j}}>$, то разрешение проблемы осуществляет функциональная зона $f_{i}$, участвующая в выполнении компонента $\mathrm{S}_{\mathrm{j}}$ стратегии ИБС. Процесс формирования сценария разрешения отдельной проблемы из $\Phi_{П С}^{1}$ предполагает выполнение ряда шагов.

1. Когнитивная структуризация. Она предполагает выделение сильных и слабых сторон, возможностей и угроз функциональной зоны $f_{i}$, характеризующих проблему и внешнюю среду. 
2. Группировка базисных концептов по группам, содержащим концепты одной категории. Выделение существенно важных концептов, задание значений концептов в лингвистической шкале.

3. Построение когнитивной карты (КК) коренной проблемы из $\Phi_{П C}^{1}$.

4. Структурный анализ КК. Он устанавливает особенности каузальных отношений между концептами КК, исследует такие свойства как:

- анализ целей на непротиворечивость;

- анализ управленческих воздействий на согласованность с целями;

- анализ эффективности интегрального воздействия управляющих концептов на целевые концепты.

5. Оценка эффективности вариантов управленческих решений по преодолению коренной проблемы из $\Phi_{П C}^{1}$.

6. Выбор эффективного сценария разрешения коренной проблемы из $\Phi_{\Pi \text { IС }}^{1}$.

После определения эффективных сценариев разрешения всех проблем из $\Phi_{П С}^{1}$, на их основе формируется сценарий преодоления текущей ПС, определяющий модель управления разрешением ПС. Для этого каждый сценарий разрешения корневой проблемы $\Phi_{П С}$ представляют в виде сетевого графика выполнения укрупненных работ. Осуществив «сшивание» (интеграцию) полученного семейства сетевых графиков в один сетевой график, получают сценарий разрешения текущей ПС, определяющий модель управления поведение ИБС.

4. Заключение. В результате исследований разработаны концептуальные и технологические основы управления ИВС, разработана процедура идентификации и когнитивного анализа проблемной ситуации, создана методика формирования сценария ее разрешения. Полученные результаты определяют предметную технологию когнитивного управления интеллектуальной бизнес системой, которая может быть использована в качестве теоретической основы при создании соответствующей информационной технологии управления. 
Гернет Н.Д., Лисицкий В.Л. Когнитивное управление интеллектуальныли..., стр. 46-55 Gernet N.D., Lisitsky V.L. Cognitive management of intellectual business..., pp. 46-55

\title{
СПИСОК ЛИТЕРАТУРЫ
}

1. Интеллектуальные системы и технологии / Советов Б.Я., Цехановский В.В, Чертовской В.Д. - М.: Академия, 2013.

2. Инновационный менеджмент в России: вопросы стратегического управления и научно-технологической безопасности. / Руководители авт. колл. В.Л. Макаров, А.Е. Варшавский. -- М.: Наука, 2004.

3. Поспелов Д.А. Ситуационное управление. Теория и практика. М.: Наука, 1987.

4. Чернавский Д.С. Синергетика и информация: Динамическая теория информации. / Предисл. и послесл. Г.Г. Малинецкого. Изд.3-е, доп. - М.: Книжный дом «ЛИБРОКОМ», 2009.

5. Гернет Н.Д., Лисиикий В.Л. Целеполагание в бизнес системах // Анализ и моделирование экономических и социальных процессов / Математика. Компьютер. Образование: Сб. научн. трудов. Выпуск 25. - М.-Ижевск: НИЦ «Регулярная и хаотическая динамика», 2018. С. 165-170.

6. Лисиикий В., Гернет Н. Прогнозирование и планирование переходных процессов в организациях. Моделирование и анализ процессов реформирования организационных систем. Издатель: LAP LAMBERT Academic Publishing. 2015.

7. Воронин А.А., Губко М.В., Мишин С.П., Новиков Д.А. Математические модели организаций. - М.: Ленанд, 2008. 360 с.

8. Саати Т. Принятие решений: метод анализа иерархий. - М.: Радио и связь. 1993.

9. Гаврилова Т.А. Базы знаний интеллектуальных систем. - СПб.: Питер. 2001.

10. Глухих И.Н. Интеллектуальные информационные системы. - СПб.: Питер. 2001.

\section{COGNITIVE MANAGEMENT OF INTELLECTUAL BUSINESS SYSTEMS: CONCEPTUAL AND TECHNOLOGICAL BASES}

\author{
Gernet N.D., Lisitsky V.L.
}

The paper proposes the subject technology of cognitive management of intelligent business systems that operate in conditions of intensive formation of the information society. The basis of cognitive management is the technology of situational management. The features of the stages of cognitive management are considered. A procedure has been developed for identifying and resolving problem situations that arise at the stage of implementation of the strategy of an intelligent business system. 